I have often found very beneficial. In this case, however, we had to apply means to relieve the immediate symptoms; and in consultation with my brother, we determined on relieving the congestion of the head by the application of leeches, whilst we endeavoured to stimulate the body by brandy, ammonia, \&c.

March 5th, 1845 .

\section{MALFORMATIONS OCCURRING IN A CHILD.}

By F. Robinson, Esq. New castle.on Tyne.

DANiEL C-_, aged nine months, a stout, healthy-looking child, was admitted into the infirmary, under the care of Sir John Fife, with a short vascular tube projecting from the umbilicus, about three-quarters of an inch in length, and the same in circumference. It is round, of an oblong shape and deep-red colour, and presents a moist, shining appearance. It emerges from the lower half of the umbilicus, which presents the usual appearance, and the base of the mass is much constricted, so as to give it somewhat the resemblance of a knuckle of intestine, where the strangulation has been recent; on looking at it, however, more closely, it is seen to differ from the latter, in presenting a granular appearance, and on being handled it feels flaccid. The tube is also slight, constricted in the middle, and at the apex there is a small orifice, from which, on a probe being introduced, it passes in a direction, directly downwards and forwards, towards the pubis; and from the limited motion that could be used with the probe laterally, the instrument seems to be contained in a prolongation of the tube.

The latter is constantly covered on its whole surface with an exudation of thin fluid, devoid of odour or colour, and a constant oozing of fluid of the same character proceeds from the orifice at the apex. The mass hangs pendulous towards the right side of the abdomen, when the child is quiet, giving the tube rather the appearance of being slightly twisted; but when the child uses any exertion in which the abdominal wall is contracted, the mas increases slightly in size and becomes of a much darker hue, rendered very apparent each time that the child alternately contracts and relaxes the abdominal muscles in crying; and at such period, instead of hanging pendulous, it projects straight out, in a direction rather upwards.

The boy is stout, has been always healthy since its birth, has never had any cough, diarrhœa, vomiting, or any affection likely to cause relaxation of the abdominal muscles. He appears to suffer no inconvenience from the tumour, and does not cry when it is handled, but slight pressure at its base is sufficient to cause it to bleed round the apex, but not from the orifice itself. Nothing abnormal can be detected by examining the abdomen.

The mother states, that the ligature which was placed round the umbilical cord came off in nine days' time, and that a small round tumour, of the same appearance as at the present time, remained projecting from the abdomen, and evidently forming the divided extremity of the cord itself. This tumour could be readily reduced into the abdominal cavity by using slight pressure with the finger, but it soon became so constricted at the point of exit from the abdomen, as to render its reduction at first difficult, and afterwards wholly impracticable. No bleeding or discharge ensued when the ligature dropped off, but about three days after a small quantity of thin fæcal matter was discharged from the orifice at the extremity of the tube, which was then first noticed. This continued, at first, every day, then every two or three days, and finally ceased altogether at the end of three months from the birth of the child.

The period when the freal matter escaped was invariably the same as when the child had an evacuation per anum. When the discharge of fæces from the tube ceased, the exudation of the colourless fluid from its surface and orifice commenced, and has continued ever since, becoming, however, rather less in quantity latterly. The fluid never resembled urine either in colour or odour, and the child never had any symptoms of suppression of urine, or any affection of the bladder. Ever since its existence, the tumour has shown a disposition to bleed when handled roughly, but not otherwise. It has gradually increased in size up to the present time. Bowels generally opened regularly, and motions of natural appearance and consistence; has had various emollient applications to the part, and has latterly been poulticing it, without, however, causing any diminution in size.

On the morning of the 25th, Sir John Fife determined to remove the mass, and commenced the operation by passing a ligature tightly round the base of the tumour, close to the abdomen; this immediately caused it to turn to a purple hue, and to exude blood at the apex. It was then excised close below the ligature, and the latter not having exerted sufficient pressure, a strong jet of arterial blood proceeded from the inside of the ex. tremity of the divided tube. This was immediately suppressed by the application of a second ligature, after a very small quantity of blood had escaped. A piece of wet lint, compress, and bandage, were then applied, and the child sent to bed. It did not appear to be at all affected by the operation.

On examining the tumour after its removal, it was found to be a tube capable of containing a quill in its cavity, and formed of a strong fibrous coat internally, lined by a thin membrane, and covered over by skin externally, a good deal of loose cellular tissue separating the two tissues. The fihres were all arranged longitudinally, with the exception of a few scattered ones that passed transversely across. Numerous small vessels were ohserved, running up to the apex of the tube, among the cellular tissue. A few minutes' maceration in tepid water rendered it quite colourless.

The mother took the child away after the operation, but returned in five days' time with it, when it was found that the part was almost well. The ligature had dropped off, and the portion of tube behind it had retracted to a level with the umbilicus, and had quite cicatrized, except a very minute space. The child was in good health, and had been so since the operation.

Much difference of opinion existed regarding the nature of the malformation. It would seem to be a portion of the umbilical cord, which, instead of withering and dropping off, became organized, but whether it communicated with any of the viscera, or terminated in a cul de sac, of course could not be determined satisfactorily.

Setting aside the latter hypothesis, the principal doubt existing was, whether the tube communicated with the intestine, formed a portion of it, or was continued down in the carity of the abdomen to the bladder, forming an abnormal connexion with the latter, and possibly communicating with the intestinal canal, at the time of birth, and for a period after. The latter appeared the more probable of the two hypotheses, as, first, its direction was in favour of that opinion; secondly, as the bladder of a child in the healthy state is known never to contain a large quantity of urine at a time, that viscus being instinctively emptied of its contents when but a small quantity is contained in it; this fact, together with the opposition afforded by gravity, would account for little or no urine being discharged by the tube, and if any, it would probably be evacuated when the child was in the horizontal posture, and perhaps asleep, and therefore it would be liable to escape the notice of the mother, especially as such applications as ointments or poultices were pretty constantly applied. Lastly, the exudation of fluid, which kept the part constantly moistened, might be accounted for by effusion of serum of the blood arising from the obstruction to the circulation in the extremity of the tube, which manifestly existed.

With regard to the supposition of the tube forming a portion of and terminating in the intestine, this, though at first consider. ation the most probable, is yet unlikely, from the straight direction of the tube; while the composition of its coats seem at least to prove that it did not form any part of the intestinal canal. Certainly, the force of gravity ard the consistence of frees might hinder the escape of any, but purgation, when used, had no effect in causing this.

Perhaps the most probable theory is, that the tube may be a continuation of the hypogastric artery, terminating in a cul de sac, and differing from the general rule in course or origin, on both. In support of this opinion the history seems to prove-if what the mother says can be relied on-that the tube was evidently a portion of the umbilical cord, and if so, the former is most likely the remains of the artery only, partially obliterated; and instead of dropping off, continuing organized, and covered with a prolongation of skin, from the margins of the umbilicus The little disturbance of the general health, and the perfect performance of the functions of the digestive and urinary apparatus seem to be also in favour of the latter opinion, as well as the rapid cure effected by the operation.

March 2nd, 1815.

CASE OF ACUTE LARYNGITIS.

By Walter Chapman, Esq. Surgeon, Norwood, Middlesex. TRACHEOTOMY.-RECOVERX.

ON Wednesday, the 19th of last February, at ten o'clock P.M., was summoned to attend Mrs. $M-$, residing in this neighbourhood. My patient was thirty-one years of age, the mother of five children, and was attended by me in her last confinement, about ten months since. Mrs. M- was an extremely thin, spare person, possessing but little physical power, and addicted (as 1 have been informed) to gin-drinking, which, however, she denied. She has generally enjoyed good health, and was suckling her infant when she became the subject of the present attack. The following is the substance of her own account of it:-She was in her usual state of health when she retired to rest on Tuesday, the 